\title{
Lexis
}

Journal in English Lexicology

HS 2 | 2010

Theoretical Approaches to Linguistic (Im)politeness

\section{Argumentation et impolitesse dans les débats politiques à caractère polémique}

Nadia Lahiani

\section{OpenEdition}

Journals

Édition électronique

URL : http://journals.openedition.org/lexis/806

DOI : $10.4000 /$ lexis.806

ISSN : 1951-6215

\section{Éditeur}

Université Jean Moulin - Lyon 3

Référence électronique

Nadia Lahiani, «Argumentation et impolitesse dans les débats politiques à caractère polémique », Lexis [En ligne], HS 2 | 2010, mis en ligne le 06 septembre 2010, consulté le 19 avril 2019. URL : http:// journals.openedition.org/lexis/806; DOI : 10.4000/lexis.806 


\title{
Argumentation et impolitesse dans les débats politiques à caractère polémique
}

\author{
Nadia Lahiani ${ }^{1}$
}

\section{Résumé}

L'argumentation a très souvent été appréhendée comme un moyen de régulation des désaccords. Nous tenterons de démontrer dans cet article que, dans les débats politiques à caractère polémique, l'argumentation peut expliciter le désaccord entre les interactants, voire, dans certains cas, trahir une impolitesse linguistique.

Mots-clés : interactions verbales - polémique - réfutation - argumentation ad hominem argumentation ad personam - F.T.A's

$* * *$

\begin{abstract}
Arguing has often been apprehended as a means of conflict settlement. In this article, we will attempt to show that in controversial political debates, arguing can explain the conflict between people interacting, or even betray a linguistic impoliteness.
\end{abstract}

Keywords: verbal interactions - controversy - refutation - ad hominem arguing - ad personam arguing - F.T.A's

\footnotetext{
${ }^{1}$ Institut Supérieur des Langues de Tunis, Tunisie : nadialahiani@gmail.com
} 


\section{Introduction}

Suite aux travaux de Palo Alto, Brown et Levinson et Kerbrat-Orecchioni, on s'est intéressé en linguistique à la politesse linguistique. Or dans les discours, et plus particulièrement dans les discours polémiques (auxquels nous nous intéressons tout particulièrement), nous remarquons assez souvent que derrière une apparente politesse, qui s'exprime linguistiquement par les modalisateurs, les atténuations, les prises en charge de l'énoncé par l'énonciateur (je suis désolé de vous dire, je m'excuse mais, il me semble que...) peuvent se cacher des violences et des agressions verbales qui s'exprimeront soit explicitement soit implicitement. Ces impolitesses vont ainsi apparaitre à différents niveaux linguistiques (argumentatif, sémantique, pragmatique).

Nous proposons, dans le cadre de cette intervention, de limiter notre analyse de l'impolitesse, au niveau argumentatif.

Nous tenons à préciser d'abord que nous nous inscrivons dans le cadre de la linguistique pragmatique et que nous appréhendons l'argumentation dans ses manifestations interactionnelles.

$\mathrm{Au}$ niveau interactionnel, l'impolitesse linguistique peut s'exprimer autant par l'argumentation sur la personne que par le biais de la réfutation. L'apparition de ces différentes formes d'attaques est relative à la nature même du discours polémique. En effet, la polémique a principalement pour cause un affrontement dû à des divergences permanentes relatives à une question. Ces divergences sont déterminées par des opinions, des prises de positions politiques, idéologiques ou autres. En ce sens le discours polémique fait partie des discours antagonistes à forte composante réfutative. Toutefois, la permanence de la confrontation et la composante réfutative ne suffisent pas à définir un discours comme étant polémique.

La polémique se distingue des autres discours ou des autres interactions antagonistes par une visée pragmatique et un cadre participatif particuliers. En effet, dans ce type d'interactions nous avons d'un côté les deux protagonistes : le Proposant et l'Opposant qui expriment des opinions qui n'acceptent pas de concession, et de l'autre coté le Tiers qui est représenté par le public, le spectateur, l'auditeur... et qui est la principale personne à convaincre.

À partir de là, les participants vont user de tous les procédés linguistiques et argumentatifs dont ils disposent, dans le but de convaincre l'auditoire de la non-validité de l'argumentation de l'interlocuteur afin de le disqualifier voire le discréditer. Cet effet perlocutoire attaché au discours polémique (discréditer l'interlocuteur et susciter l'adhésion du public) constitue un terrain propice à la manifestation de l'impolitesse linguistique en tant que transgression des règles de politesse.

Nous partons donc de l'hypothèse que dans les discours polémiques, c'est souvent la transgression des règles de politesse linguistique qui domine et qui structure le discours dans le but d'atteindre un objectif: convaincre l'auditoire du bien-fondé de ses propos tout en discréditant l'interlocuteur. Nous vérifierons cette hypothèse en démontrant dans un premier temps que l'opposition et le rejet de l'argumentation de l'interlocuteur peut s'exprimer par le biais de la réfutation. Nous nous intéresserons ensuite, aux cas où la réfutation constitue une attaque à la personne de l'interlocuteur. Enfin, nous nous pencherons sur les cas où la violence des termes par lesquels s'exprime la réfutation peut constituer une attaque contre la personne qui s'apparente à l'injure.

L'objectif de cet article est de démontrer, à partir d'exemples extraits de débats politiques, que dans les discours polémiques, les argumentations (contrairement à l'analyse qu'ont font Aristote ou Perelman) ne sont pas un moyen de réguler le désaccord et de réparer les menaces à la face, mais vont, au contraire, contribuer à expliciter la divergence et 
l'opposition entre les interlocuteurs. La visée pragmatique du discours polémique favoriserait l'interprétation de ces argumentations, comme étant des impolitesses linguistiques

\section{La réfutation}

L'apparition de la polémique est relative à une divergence d'opinion sur une question déterminée. Ce désaccord est marqué au niveau argumentatif par différentes procédures à travers lesquelles s'effectue l'acte de réfutation. La réfutation présuppose l'existence d'un discours explicite ou implicite auquel elle s'oppose. En tant qu' « acte réactif d'opposition », la réfutation de ce qui est dit ou de ce qui a été dit peut porter sur différents éléments de l'énonciation.

En effet, dans le contexte d'une polémique, un locuteur peut réfuter autant l'argumentation qu'un élément de l'argumentation de son interlocuteur. Ainsi, si un Proposant énonce («Je travaille beaucoup et je réussirai »), l'Opposant peut réfuter son énoncé en lui opposant (« Ce n'est pas la quantité de travail qui assure la réussite »). Ce qui est réfuté dans cet énoncé c'est la pertinence de l'argument «beaucoup » et donc la conclusion qui en découle.

Cette réfutation repose sur la loi de passage qui stipule que « ce n'est pas la quantité de travail qui compte mais c'est la qualité ». Ce «topoï» permet à l'Opposant de réfuter l'énoncé «Je travaille beaucoup » orienté vers la conclusion $C$ «je réussirai » et d'orienter l'argumentation vers la conclusion non $C$ « tu ne réussiras pas » en se basant sur le topoï « $\mathrm{Ce}$ n'est pas la quantité de travail qui assure la réussite ».

Mais la présence d'une réfutation, comme c'est le cas dans cet exemple, bien qu'elle constitue une transgression des règles de politesse, n'implique pas une impolitesse. La politesse linguistique consiste à ne pas produire un acte qui menacerait soit la face positive, qui correspond selon C. Kerbrat-Orecchioni [1992:168]

$\mathrm{Au}$ narcissisme et à l'ensemble des images valorisantes que les interlocuteurs construisent et tentent d'imposer d'eux-mêmes dans l'interaction

Soit la face négative définie par C. Kerbrat-Orecchioni [1992 : 167] comme étant :

Le territoire corporel, spatial, temporel, bien et réserves, matérielles ou cognitives de l'interlocuteur

Toute menace à l'une de ces faces constitue un FTA ${ }^{2}$. Contredire son interlocuteur et réfuter son énoncé constitue une menace à sa face positive, puisque par cet acte le locuteur «met en péril le narcissisme » [Kerbrat-Orecchioni 1992 : 170] de son interlocuteur.

Mais la menace à la face n'est pas une impolitesse linguistique. Pour identifier une impolitesse linguistique dans un énoncé, il est nécessaire de tenir compte d'éléments extralinguistiques tels que le contexte d'énonciation, le statut et la relation entre les interactants, la visée du discours, etc.

Autrement dit, pour qu'il y ait impolitesse linguistique, il faut que le locuteur vise, par le biais de son énonciation, à accomplir un acte perlocutoire tel que discréditer, disqualifier, rabaisser, insulter, injurier ou autres, son interlocuteur. Or dans les débats politiques à caractère polémique, le locuteur vise assez souvent à discréditer son interlocuteur en recourant à la réfutation.

\footnotetext{
${ }^{2}$ Terme de Brown et Levinson.
} 


\subsection{La réfutation comme acte d'opposition}

Dans le cadre d'un débat politique à caractère polémique, il arrive que la réfutation constitue une attaque autant à la face positive qu'à la face négative. C'est ce que nous relevons dans cet échange. ${ }^{3}$

MLP : Plein de choses si vous me laissez parler vous avez dit que N. Sarkozy a beaucoup parlé pendant la campagne présidentielle d'immigration oui

EB : et agit

MLP : non monsieur il n'a strictement rien fait

Dans cet échange EB ajoute à l'énoncé de MLP, «N.S a parlé d'immigration », une information "agit», qui oriente l'argumentation vers une conclusion positive : " il tient ses promesses et il fait ce qu'il dit». Or, par l'acte d'opposition «non il n'a strictement rien fait », son interlocutrice réfute un élément de son argumentation, la prémisse spécifique : «il a agit ». Cette réfutation lui permet de casser la relation entre les verbes « dire » et « agir » qui sont en progression positive, et de modifier l'orientation de l'argumentation de son interlocuteur, puisqu'elle transforme la conclusion positive $\mathrm{C}$, « il fait ce qu'il dit », en une conclusion non $\mathrm{C}$, « il ne tient pas ses promesses ».

P g : Vous avez dit qu'il a beaucoup parlé

$\mathrm{P}$ s : Mais il n'a strictement rien fait

$\mathrm{C}$ : Donc il ne tient pas ses promesses et il ne fait pas ce qu'il dit

Cette réfutation constitue une menace à la face positive de l'interlocuteur, puisqu'elle conteste la véracité de l'information apportée par son énoncé. Mais elle constitue en même temps une menace pour la face négative d'EB. En effet, dans cet échange MLP, remet en question la sincérité des promesses électorales de N. Sarkozy. Or, EB est présent en tant que ministre de ce Président. Donc, en s'attaquant à N. Sarkozy, la locutrice affecte une des « réserves matérielles de l'individu » [Kerbrat-Orecchioni [1992: 167], la personne qu'il représente et qu'il défend et qui fait par conséquent partie de son territoire. Comme le code de l'interaction fait passer le statut du locuteur avant sa personne, en visant à discréditer le discours électoral de NS, elle affecte indirectement la personne d'EB.

\subsection{Une réfutation pour disqualifier}

Il arrive que la réfutation, ne porte pas sur un élément de l'argumentation, mais qu'elle s'effectue par le biais du rejet de l'argumentation de l'interlocuteur. Ce rejet peut s'accomplir par l'expression d'un jugement négatif émis sur le discours du Proposant. Nous retrouvons ce type de réfutation dans cet extrait ${ }^{4}$ :

NM : donc je n'oublie pas que N. Sarkozy était maire de Neuilly président du conseil général des Hauts-de-Seine je n'oublie pas qu'un certain Bernard Bled qui était mouillé dans quelques affaires avec M. Tiberi a été directeur général de

\footnotetext{
${ }^{3}$ Extrait du débat $A$ vous de juger du 14/10/2009, qui a opposé Marine le Pen (noté MLP) à E. Besson (noté EB) sur le thème de l'identité nationale.

${ }^{4}$ Cet échange, qui a opposé Noel Mamère (noté NM) à Frédéric Lefebvre (noté FL) au sujet de l'élection de Jean Sarkozy à la présidence de l'EPAD, est extrait du débat télévisé $A$ vous de juger du 22/10/2009.
} 
l'EPAD et je n'oublie pas que l'EPAD c'est effectivement un lieu très intéressant pour ceux qui s'intéressent au développement des Hauts-de-Seine

FL : Ça s'appelle de la calomnie

Ce que réfute FL dans son acte de langage c'est la conclusion sous-entendue par l'affirmation de NM : «l'élection de JS n'est pas due à son mérite mais à un favoritisme qui se justifie par le statut de son père ».

Toutefois, cette réfutation ne procède pas par le rejet d'un élément de l'argumentation, mais par la disqualification de toute l'argumentation de l'interlocuteur. Cette disqualification est relative aux sèmes négatifs « diffamations, allégations, mensonges, accusations » associés à la connotation du substantif «calomnie » qui définit le discours de NM. Ces qualifications négatives du discours de l'interlocuteur constituent indirectement une qualification négative de la personne de l'interlocuteur, et orientent cette attaque vers une conclusion contre la personne « vous êtes un menteur».

«La maxime argumentative du dire» [Plantin 1990: 255] nous fournit une loi de passage de l'argument «Ça s'appelle de la calomnie»vers la conclusion «vous êtes un menteur ». Cette règle topique stipule que : "si la parole de L est moins, alors L est moins » [Plantin $1990: 255]$.

Donc, à partir du moment où le substantif «calomnie» qualifie la parole de l'interlocuteur négativement, les lieux communs qu'on peut dériver de cette maxime argumentative : "On reconnait un arbre à ses fruits, dis-moi ce que tu fais je te dis qui tu es... » vont constituer une loi de passage vers la conclusion « tu es un menteur ».

Par ailleurs cette attaque a une autre particularité : elle affecte l'éthos de l'interlocuteur :

L'éthos représente les qualités liées à la personne de l'orateur, c'est l'image qu'il donne de lui auprès du public. [Robrieux 1993 : 18]

Dans un débat, et à plus forte raison dans un débat politique, chaque interactant cherche à donner une image positive qui inspire la confiance et qui lui permet d'emporter l'adhésion du public. Or, en disqualifiant les arguments et la personne de l'interlocuteur, le locuteur vise d'une part à donner de lui une image «rebutante» [Ducrot 1984: 201], et d'autre part à pousser l'auditoire à ne pas avoir foi en ce qu'il a dit, dit ou pourrait dire.

Recourir aux évaluations négatives pour réfuter l'argumentation de l'interlocuteur constitue donc, dans ce cas, une impolitesse linguistique. Cette impolitesse s'explique par le fait qu'au niveau pragmatique la réfutation vise la disqualification du discours du Proposant. En effet, le statut interactionnel de la réfutation lui concède, généralement, un caractère violent « réfuter c'est abattre » [Plantin 2002: 405] et brutal « celui qui réfute prétend clore le débat » [Plantin 2002: 405]. Ce qui justifie sa récurrence dans les discours polémiques et contribue à la classer, dans le cadre de ce type de discours, parmi les modes d'expression des impolitesses linguistiques.

\section{L'argumentation contre la personne}

L'argumentation contre la personne, appelée argumentation ad hominem procède par une opposition entre un "dire » et un autre "dire». Mais elle peut aussi procéder par une « opposition entre "le dire" et "le faire" » [Gauthier 1995 : 174]. Dans ce cas, l'argumentation est de nature pragmatique puisqu'elle repose sur les actes de la personne attaquée. Pour cette raison, J-J. Robrieux [1993 : 142] la classe parmi les argumentations ad personam : 
L'argument ad personam est celui qui met en évidence l'opposition entre ce que l'on sait d'une personne et ce qu'elle a dit ou fait. C'est donc une manière de disqualifier l'adversaire en confrontant deux observations, celle de sa personne et celle de ses actes ou de ses affirmations.

\subsection{L'argumentation ad personam}

La particularité de ce type d'argumentation est qu'elle permet de discréditer la position défendue par l'interlocuteur en donnant « la preuve » [Robrieux 1993 : 142], par les faits, de son incohérence ou de sa malhonnêteté. Nous retrouvons ce type d'argument dans l'échange suivant ${ }^{5}$ :

EB : Oui mais je vais essayer de alors je vous trouve outrecuidante vous préférez c'est plus joli que gonflée allons y alors je vous trouve outrecuidante parce que vous donnez en permanence des leçons sur l'immigration clandestine l'immigration irrégulière etc. mais vous vous trompez les français parce que vous avez été nommée élue plus exactement excusez moi avez été élue députée européenne vous n'êtes quasiment jamais

MLP : (C'est faux monsieur)

EB : (vous êtes de 2004) à 2009 la députée française européenne la plus absente $[\ldots]$

EB : mais surtout lorsqu'ont été discutées les directives les plus importantes dont ce qu'on appelle la directive retour qui traitait de la lutte conte l'immigration irrégulière et que nous allons prochainement transposer je vous l'annonce $\mathrm{AC}$ en février je dépo je présenterai au conseil des ministres une loi dite de transposition de cette directive qui va donc lutter davantage contre l'immigration irrégulière et qui va prendre des initiatives vous n'étiez pas madame à la commission vous n'avez pas dit un mot vous n'avez pas intervenu donc en France vous venez dire $[\ldots]$

Dans cet échange, EB met en contradiction l'image que MLP véhicule à travers ses différents propos et discours (vous donnez en permanence des leçons sur l'immigration clandestine l'immigration irrégulière, etc.) et ses actes (vous n'étiez pas madame à la commission vous n'avez pas dit un mot vous n'avez pas intervenu donc en France vous venez dire). Les différents éléments de son argumentation se présentent comme suit :

P g : Quand on a la volonté de lutter contre l'immigration clandestine, on assiste et on participe activement aux débats qui s'y rapportent.

P s : Or vous n'allez pas à la commission et vous n'êtes pas intervenue pour défendre cette position.

$\mathrm{C}$ : Donc vous vous désintéressez du sujet et vous trompez les français en affirmant le contraire.

Dans cette argumentation, le locuteur confronte les actions de son interlocutrice à ses discours, et relève la contradiction qui en découle. En relevant cette opposition, d'une part, le locuteur donne la preuve que les actes sont incohérents par rapport aux discours et aux principes défendus par MLP. D'autre part, il remet en question son intégrité idéologique et la

\footnotetext{
${ }^{5}$ Cet échange est extrait du débat télévisé $A$ vous de juger du 14/10/2010 qui portait sur le thème de l'identité nationale et qui a opposé Eric Besson à Marine Le Pen.
} 
sincérité de son engagement politique. Ce qui justifie la conclusion «vous trompez les Français ».

Mais ce qui attire particulièrement notre attention dans cette argumentation, c'est que généralement, par souci de politesse linguistique, la prémisse ou la conclusion qui contient l'attaque la plus virulente est omise. Par contre, dans ce discours le locuteur explicite les prémisses et la conclusion de son argumentation. Ce qui n'autorise aucun doute quand à la visée disqualifiante de son discours, et l'impolitesse linguistique qui en découle.

En effet, dans cette argumentation la prémisse générique et la prémisse spécifique constituent déjà une menace à la face de l'interlocutrice, dans la mesure où le simple fait de les confronter met en évidence l'opposition entre les actions et les discours. La conclusion « vous trompez les Français » aurait pu donc être déduite de l'argumentation. Mais à partir du moment que cette conclusion est énoncée, l'attaque à la face et la visée disqualifiante de ce discours deviennent directes et explicites.

\subsection{Discréditer le statut de l'interlocuteur}

Dans le cadre d'un débat polémique, toutes les attaques sont autorisées pour atteindre son but. Même celles qui consistent à détourner l'argumentation du sujet du débat et de s'en prendre directement à la personne de son interlocuteur.

MLP : excuse moi je trouve que le Parti Socialiste en l'occurrence est au dessous de tout je pense que heuu ça démontre d'ailleurs que le Front National est le seul réel opposant à N. Sarkozy mais ce n'est pas très grave M. Besson je pense que vous êtes capable de faire en même temps le socialiste et le responsable de $1^{\prime} \mathrm{UMP}^{6}$

Dans cet énoncé, MLP attaque EB en s'en prenant à sa personne et plus précisément à son statut, dans la mesure où EB est présent sur le plateau en tant que représentant de l'UMP. Cette attaque se fonde sur les connaissances mutuelles des interlocuteurs. MLP sait, comme tous les Français d'ailleurs, qu'EB était au Parti Socialiste jusqu'aux primaires des élections présidentielles de 2007. Mais il est présent sur le plateau en tant que représentant de l'UMP et donc de la droite. Or, passer d'un parti de gauche qu'il a défendu pendant 15 ans, et se présenter sur le plateau pour défendre les actions d'un gouvernement de la Droite qu'il représente constitue pour le moins une anomalie politique.

L'attaque de la locutrice va porter sur ce revirement de position politique et sur l'incompatibilité idéologique qui lui est associée. En effet, la position du sujet est relative à «la Formation Discursive» [Courtine 1981: 35] à laquelle il s'identifie ainsi qu'aux domaines de mémoire et aux préconstruits idéologiques qui lui sont associés. Or la Droite et la Gauche relèvent de deux formations discursives antagonistes, et défendent par conséquent des positions politiques, théoriquement, incompatibles (du moins dans leurs discours officiels). En ce sens, EB se trouve dans une position où il doit défendre un gouvernement qui prône une idéologie contraire et incompatible avec celle du parti auquel il avait longtemps appartenu. Et c'est sur ce point qu'il est attaqué.

En l'attaquant sur ce revirement de position politique et idéologique, la locutrice relève une opposition entre deux situations, positions et choix politiques, qui prouve l'incohérence intellectuelle et idéologique du locuteur, voire sa malhonnêteté, dans la mesure où elle sousentend qu'il sacrifie ses convictions et son intégrité politique et intellectuelle à son ambition personnelle.

\footnotetext{
${ }^{6}$ Extrait du débat $A$ vous de juger du 14/10/2010.
} 
Cette preuve permet à MLP de décrédibiliser le locuteur, et par conséquent les éventuels arguments qu'il pourrait développer.

\section{La réfutation dans la polémique}

Nous avons expliqué dans la première partie de cet article que la réfutation était un acte argumentatif d'opposition à un élément de l'énonciation. Mais, lorsque dans le cadre d'un débat polémique les agressions du proposant sont explicites et virulentes, l'opposant peut réfuter l'énoncé de son interlocuteur violemment, au risque de ne plus respecter les règles de politesse linguistique.

\subsection{Réfutation du statut du discours}

L'agressivité de l'attaque peut relever de la signification des termes utilisés et de la visée argumentative de l'acte de langage effectué. Nous retrouvons ce type de réfutation dans l'échange suivant : ${ }^{7}$

EB : laissez-moi m'exprimer puis après vous répondrez sur le débat sur l'identité nationale vous dites en gros vous avez l'avez dit sur une émission de télévision les Français sont de race blanche

MLP : Je n'ai absolument M. Besson jamais dit cela

EB : (je vous je vous) le prouverai c'était sur («c'est-à-dire» et je renvoie les internautes à)

MLP : (Je n'ai jamais dit cela) (ah non je n'ai jamais dit ça là vous mentez mais vous mentez totalement monsieur) J'ai cité une citation du Général de Gaulle

Dans cet échange MLP réfute l'information «les Français sont de race blanche » communiquée par EB. Mais l'acte de langage "vous mentez, vous mentez totalement» n'implique pas uniquement une réfutation de ce qui est dit. En employant le verbe «mentir » qui appartient à la classe des verbes « intrinsèquement subjectifs » [Kerbrat-Orecchioni 1980 : 101], la locutrice porte une évaluation négative "sur le procès dénoté (et par ricochet sur l'agent qui en porte la responsabilité) » [Kerbrat-Orecchioni $1980: 101]$.

Ce qui signifie que le verbe «mentir» permet à la locutrice de poser que son interlocuteur introduit volontairement une information fausse, et de présupposer que le sujet de cette information " est un menteur ». Le rejet de cette information prend alors la forme d'une accusation injurieuse, "vous mentez », par laquelle la locutrice vise autant à rejeter l'énoncé de l'interlocuteur, qu'à disqualifier sa personne. Le caractère injurieux et la visée pragmatique de la proposition « vous mentez » constituent une impolitesse linguistique.

\section{Conclusion}

A partir de cette analyse de l'impolitesse exprimée au niveau argumentatif nous avons remarqué que la visée argumentative et pragmatique du discours politique à caractère polémique favorise l'apparition de la réfutation et des argumentations contre la personne. Ces argumentations mettent en péril le narcissisme de l'interlocuteur et menacent sa face.

\footnotetext{
${ }^{7}$ Extrait du débat $A$ vous de juger du 14/10/2010
} 
Toutefois, une menace à la face ne constitue pas nécessairement une impolitesse linguistique. L'impolitesse est un cas particulier de FTA, qui est relative et dépendante de la visée pragmatique du discours en général et du discours politique à caractère polémique en particulier, en l'occurrence discréditer et disqualifier l'interlocuteur et son discours.

\section{Bibliographie}

Charaudeau Patrick \& Maingueneau Dominique, Dictionnaire d'analyse de discours Paris, Seuil, 2002.

COURTINE Jean-Jacques, «Quelques problèmes théoriques et méthodologiques en analyse du discours, à propos du discours communiste adressé aux chrétiens ", in Langages 64 : « Analyse du discours politique », Larousse, Paris, Juin 1981 : 9-47.

Ducrot Oswald, Le dire et le dit, Paris, Minuit, 1984.

GAUTHIER Gilles, "L'argumentation périphérique dans la communication politique ", in Argumentation et rhétorique II, Cnrs édition, Hermès 16, Cognition, Communication, Politique, Paris, 1995 : 167-185.

GOFFMAN Erving, Les rites d'interaction, traduit de l'anglais par Alain Kihm, Paris, Minuit, (1967) 1974.

Kerbrat-Orecchioni Catherine, L'énonciation. De la subjectivité dans le langage, Paris, Armand Colin, 1980.

Kerbrat-OrecChioni Catherine, Les interactions verbales, Tome II, Paris, Armand Colin, 1992.

Plantin Christian, Essais sur l'Argumentation, Paris, Kimé, 1990.

Plantin Christian, "La réfutation », in Charaudeau P. \& Maingueneau D., Dictionnaire d'analyse de discours, Seuil, Paris, 2002 : 405.

RoBRIEUX Jean-Jacques, Eléments de rhétorique et d'argumentation, Paris, Dunod, 1993. 